CUBO, A Mathematical Journal

Vol.22, $\mathrm{N}^{\circ} 02,(273-289)$. August 2020

Received: 11 September, 2019 | Accepted: 27 July, 2020

\title{
Fixed point theorems on cone $S$-metric spaces using implicit relation
}

\author{
G. S. SAluja \\ Department of Mathematics, \\ Govt. Kaktiya P. G., College Jagdalpur, \\ Jagdalpur - 494001 (C.G.), India. \\ saluja1963@gmail.com
}

\begin{abstract}
In this paper, we establish some fixed point theorems in the framework of cone $S$-metric spaces using implicit relation. Our results extend, unify and generalize several results from the current existing literature. Especially, they extend the corresponding results of Sedghi and Dung [24] to the setting of complete cone $S$-metric spaces.

\section{RESUMEN}

En este artículo, establecemos algunos teoremas de punto fijo en el marco de espacios $S$-métricos del cono usando una relación implícita. Nuestros resultados extienden, unifican y generalizan diversos resultados de la literatura actual existente. Especialmente, extienden los resultados correspondientes de Sedghi y Dung [24] en el contexto de espacios $S$-métricos de cono completo.
\end{abstract}

Keywords and Phrases: Fixed point, implicit relation, cone $S$-metric space, cone.

2020 AMS Mathematics Subject Classification: 47H10, 54H25. 


\section{Introduction and Preliminaries}

In 2007, Huang and Zhang [8] introduced the concept of cone metric spaces as a generalization of metric spaces by replacing the set of real numbers by a general Banach space $E$ which is partially ordered with respect to a cone $P \subset E$ and establish some fixed point theorems for contractive mappings in normal cone metric spaces.

In 2012, Sedghi et al. 23] introduced the concept of $S$-metric space which is different from other space and proved fixed point theorems in $S$-metric space. They also give some examples of $S$-metric space which shows that $S$-metric space is different from other spaces.

In 2016, Rahman and Sarwar [20] have discussed the fixed point results of Altman integral type mappings in $S$-metric spaces and in the same year Ozgur and Tas 14 have studied new contractive conditions of integral type in complete $S$-spaces.

Recently, Dhamodharan and Krishnakumar [6] introduced the concept of cone $S$-metric space and proved some fixed point theorems using various contractive conditions in the above said space.

Due to great importance of the fixed point theory, it is immensely interesting to study fixed point theorems on different concepts. Many authors studied the fixed points for mappings satisfying contractive conditions in complete $S$-metric spaces (see, e.g., [6, 11, 13, 14, 20, 23, 25, 26]) and others).

Popa [15] and [16], on the other hand, considered an implicit contraction type condition instead of the usual explicit condition. This direction of research produced a consistent literature on fixed point and common fixed point theorems in various ambient spaces. For more details see [1, 2, 3, 9, 17, 18, 19, 24.

Motivated and inspired by Popa [15, 16], Sedghi and Dung [24] and others, this paper is aimed to study and establish some fixed point theorems in the setting of complete cone $S$-metric spaces under implicit contractive condition which is used in [24. Following the current literature there is ample vicinity to explore and improve this new avenue of research area. Here, we prove an important result of cone $S$-metric space and then obtain some classical fixed point theorems as corollaries, for example, Banach's contraction mapping principle, Kannan's fixed point theorem, Chatterjae's fixed point theorem, Reich fixed point theorem and Ćirić's fixed point theorem in this setting. Our results extend and generalize several results from the existing literature, especially, the results of Sedghi and Dung 24] from complete $S$-metric spaces to the setting of complete cone $S$-metric spaces.

The present work is to encouraged by its possible application, especially in discrete models for numerical analysis, where iterative schemes are extensively used due to their versatility for computer simulation. These models play an important role in applied mathematics. 
We need the following definitions and lemmas in the sequel.

Definition 1. ([8]) Let $E$ be a real Banach space. A subset $P$ of $E$ is called a cone whenever the following conditions hold:

( $\left.c_{1}\right) P$ is closed, nonempty and $P \neq\{0\}$;

$\left(c_{2}\right) a, b \in R, a, b \geq 0$ and $x, y \in P$ imply $a x+b y \in P$

$\left(c_{3}\right) P \cap(-P)=\{0\}$.

Given a cone $P \subset E$, we define a partial ordering $\leq$ in $E$ with respect to $P$ by $x \leq y$ if and only if $y-x \in P$. We shall write $x<y$ to indicate that $x \leq y$ but $x \neq y$, while $x \ll y$ will stand for $y-x \in P^{0}$, where $P^{0}$ stands for the interior of $P$. If $P^{0} \neq \emptyset$ then $P$ is called a solid cone (see [28]).

There exist two kinds of cones- normal (with the normal constant $K$ ) and non-normal ones ([7]).

Let $E$ be a real Banach space, $P \subset E$ a cone and $\leq$ partial ordering defined by $P$. Then $P$ is called normal if there is a number $K>0$ such that for all $x, y \in P$,

$$
0 \leq x \leq y \text { imply }\|x\| \leq K\|y\|
$$

or equivalently, if $(\forall n) x_{n} \leq y_{n} \leq z_{n}$ and

$$
\lim _{n \rightarrow \infty} x_{n}=\lim _{n \rightarrow \infty} z_{n}=x \text { imply } \lim _{n \rightarrow \infty} y_{n}=x .
$$

The least positive number $K$ satisfying (1.1) is called the normal constant of $P$.

The cone $P$ is called regular if every increasing sequence which is bounded from above is convergent, that is, if $\left\{x_{n}\right\}$ is a sequence such that $x_{1} \leq x_{2} \leq \cdots \leq x_{n} \leq \cdots \leq y$ for some $y \in E$, then there is $x \in E$ such that $\left\|x_{n}-x\right\| \rightarrow 0$ as $n \rightarrow \infty$. Equivalently, the cone $P$ is regular if and only if every decreasing sequence which is bounded from below is convergent. It is well known that a regular cone is a normal cone. Suppose $E$ is a Banach space, $P$ is a cone in $E$ with $\operatorname{int}(P) \neq \emptyset$ and $\leq$ is partial ordering in $E$ with respect to $P$.

Example 1. ([12]) Let $K>1$ be given. Consider the real vector space

$$
E=\left\{a x+b: a, b \in R ; x \in\left[1-\frac{1}{K}, 1\right]\right\}
$$

with supremum norm and the cone

$$
P=\{a x+b \in E: a \geq 0, b \geq 0\}
$$

in $E$. The cone $P$ is regular and so normal. 
Definition 2. ([8, [29]) Let $X$ be a nonempty set. Suppose that the mapping $d: X \times X \rightarrow E$ satisfies:

$\left(C M_{1}\right) 0 \leq d(x, y)$ for all $x, y \in X$ with $x \neq y$ and $d(x, y)=0 \Leftrightarrow x=y$;

$\left(C M_{2}\right) d(x, y)=d(y, x)$ for all $x, y \in X$;

$\left(C M_{3}\right) d(x, y) \leq d(x, z)+d(z, y)$ for all $x, y, z \in X$.

Then $d$ is called a cone metric [8] on $X$ and $(X, d)$ is called a cone metric space [8] or simply $C M S$.

The concept of a cone metric space is more general than that of a metric space, because each metric space is a cone metric space where $E=\mathbb{R}$ and $P=[0,+\infty)$.

Lemma 1. ([22]) Every regular cone is normal.

Example 2. ([8]) Let $E=\mathbb{R}^{2}, P=\left\{(x, y) \in \mathbb{R}^{2}: x \geq 0, y \geq 0\right\}, X=\mathbb{R}$ and $d: X \times X \rightarrow E$ defined by $d(x, y)=(|x-y|, \alpha|x-y|)$, where $\alpha \geq 0$ is a constant. Then $(X, d)$ is a cone metric space with normal cone $P$ where $K=1$.

Clearly, the above example shows that the class of cone metric spaces contains the class of metric spaces.

Definition 3. ([23, 14]) Let $X$ be a nonempty set and $S: X^{3} \rightarrow[0, \infty)$ be a function satisfying the following conditions for all $x, y, z, t \in X$ :

$\left(S M_{1}\right) S(x, y, z) \geq 0$

$\left(S M_{2}\right) S(x, y, z)=0$ if and only if $x=y=z$;

$\left(S M_{3}\right) S(x, y, z) \leq S(x, x, t)+S(y, y, t)+S(z, z, t)$.

Then the function $S$ is called an $S$-metric on $X$ and the pair $(X, S)$ is called an $S$-metric space or simply SMS.

Example 3. (27]) Let $X$ be a nonempty set and d be the ordinary metric on $X$. Then $S(x, y, z)=$ $d(x, z)+d(y, z)$ is an $S$-metric on $X$.

Example 4. (23]) Let $X=\mathbb{R}^{n}$ and $\|\cdot\|$ a norm on $X$, then $S(x, y, z)=\|y+z-2 x\|+\|y-z\|$ is an $S$-metric on $X$.

Example 5. ([23]) Let $X=\mathbb{R}^{n}$ and $\|\cdot\|$ a norm on $X$, then $S(x, y, z)=\|x-z\|+\|y-z\|$ is an $S$-metric on $X$.

Example 6. (24]) Let $X=\mathbb{R}$ be the real line. Then $S(x, y, z)=\|x-z\|+\|y-z\|$ for all $x, y, z \in \mathbb{R}$ is an $S$-metric on $X$. This $S$-metric on $X$ is called the usual $S$-metric on $X$. 
Definition 4. ([6]) Suppose that $E$ is a real Banach space, $P$ is a cone in $E$ with int $P \neq \emptyset$ and $\leq$ is partial ordering with respect to $P$. Let $X$ be a nonempty set and let the function $S: X^{3} \rightarrow E$ satisfy the following conditions:

$\left(C S M_{1}\right) S(x, y, z) \geq 0$

$\left(C S M_{2}\right) S(x, y, z)=0$ if and only if $x=y=z$;

$\left(C S M_{3}\right) S(x, y, z) \leq S(x, x, a)+S(y, y, a)+S(z, z, a), \forall x, y, z, a \in X$.

Then the function $S$ is called a cone $S$-metric on $X$ and the pair $(X, S)$ is called a cone $S$-metric space or simply CSMS.

Example 7. ([6] $]$ ) Let $E=\mathbb{R}^{2}, P=\left\{(x, y) \in \mathbb{R}^{2}: x \geq 0, y \geq 0\right\}, X=\mathbb{R}$ and $d$ be the ordinary metric on $X$. Then the function $S: X^{3} \rightarrow E$ defined by $S(x, y, z)=(d(x, z)+d(y, z), \alpha(d(x, z)+$ $d(y, z)))$, where $\alpha>0$ is a cone $S$-metric on $X$.

Lemma 2. ([[]]) Let $(X, S)$ be a cone $S$-metric space. Then we have $S(x, x, y)=S(y, y, x)$.

Definition 5. ([6]) Let $(X, S)$ be a cone $S$-metric space.

(i) A sequence $\left\{u_{n}\right\}$ in $X$ converges to $u$ if and only if $S\left(u_{n}, u_{n}, u\right) \rightarrow 0$ as $n \rightarrow \infty$, that is, there exists $n_{0} \in N$ such that for all $n \geq n_{0}, S\left(u_{n}, u_{n}, u\right) \ll c$ for each $c \in E, 0 \ll c$. We denote this by $\lim _{n \rightarrow \infty} u_{n}=u$ or $\lim _{n \rightarrow \infty} S\left(u_{n}, u_{n}, u\right)=0$.

(ii) A sequence $\left\{u_{n}\right\}$ in $X$ is called a Cauchy sequence if $S\left(u_{n}, u_{n}, u_{m}\right) \rightarrow 0$ as $n, m \rightarrow \infty$, that is, there exists $n_{0} \in N$ such that for all $n, m \geq n_{0}, S\left(u_{n}, u_{n}, u_{m}\right) \ll c$ for each $c \in E, 0 \ll c$.

(iii) The cone $S$-metric space $(X, S)$ is called complete if every Cauchy sequence is convergent.

In the following lemma, we see the relationship between a cone metric and a cone $S$-metric.

Lemma 3. ([G]) Let $(X, d)$ be a cone metric space. Then, the following properties are satisfied:

(1) $S(u, v, z)=d(u, z)+d(v, z)$ for all $u, v, z \in X$, is a cone $S$-metric on $X$.

(2) $u_{n} \rightarrow u$ in $(X, d)$ if and only if $u_{n} \rightarrow u$ in $\left(X, S_{d}\right)$.

(3) $\left\{u_{n}\right\}$ is Cauchy in $(X, d)$ if and only if $\left\{u_{n}\right\}$ is Cauchy in $\left(X, S_{d}\right)$.

(4) $(X, d)$ is complete if and only if $\left(X, S_{d}\right)$ is complete.

Lemma 4. (24) Let $f: X \rightarrow Y$ be a map from an $S$-metric space $X$ to an $S$-metric space $Y$. Then $f$ is continuous at $x \in X$ if and only if $f\left(x_{n}\right) \rightarrow f(x)$ whenever $x_{n} \rightarrow x$.

Now, we introduce an implicit relation to investigate some fixed point theorems on cone $S$ metric spaces. Let $\psi$ be the family of all continuous functions of five variables $\phi: \mathbb{R}_{+}^{5} \rightarrow \mathbb{R}_{+}$. For some $k \in[0,1)$, we consider the following conditions. 
$\left(A_{1}\right)$ For all $x, y, z \in \mathbb{R}_{+}$, if $y \leq \phi(x, x, y, z, 0)$ with $z \leq 2 x+y$, then $y \leq k x$.

$\left(A_{2}\right)$ For all $y \in \mathbb{R}_{+}$, if $y \leq \phi(y, 0,0, y, y)$, then $y=0$.

$\left(A_{3}\right)$ If $x_{i} \leq y_{i}+z_{i}$ for all $x_{i}, y_{i}, z_{i} \in \mathbb{R}_{+}, i \leq 5$, then

$$
\phi\left(x_{1}, \ldots, x_{5}\right) \leq \phi\left(y_{1}, \ldots, y_{5}\right)+\phi\left(z_{1}, \ldots, z_{5}\right)
$$

Moreover, for all $y \in X, \phi(0,0,2 y, y, 0) \leq k y$.

Remark 1. Note that the coefficient $k$ in conditions $\left(A_{1}\right)$ and $\left(A_{3}\right)$ may be different, for example, $k_{1}$ and $k_{3}$ respectively. But we may assume that they are equal by taking $k=\max \left\{k_{1}, k_{3}\right\}$.

\section{Main Results}

In this section, we shall prove some fixed point theorems using implicit relation in the setting of cone $S$-metric spaces.

Theorem 1. Let $T$ be a self-map on a complete cone $S$-metric space $(X, S)$, $P$ be a normal cone with normal constant $K$ and

$$
\begin{gathered}
S(T x, T x, T y) \leq \phi(S(x, x, y), S(x, x, T x), S(y, y, T y) \\
S(x, x, T y), S(y, y, T x))
\end{gathered}
$$

for all $x, y \in X$ and some $\phi \in \psi$. Then we have

(1) If $\phi$ satisfies the condition $\left(A_{1}\right)$, then $T$ has a fixed point. Moreover, for any $x_{0} \in X$ and the fixed point $x$, we have

$$
S\left(T x_{n}, T x_{n}, x\right) \leq\left(\frac{2 k^{n}}{1-k}\right) S\left(x_{0}, x_{0}, T x_{0}\right)
$$

(2) If $\phi$ satisfies the condition $\left(A_{2}\right)$ and $T$ has a fixed point, then the fixed point is unique.

(3) If $\phi$ satisfies the condition $\left(A_{3}\right)$ and $T$ has a fixed point $x$, then $T$ is continuous at $x$. 
Proof. (1) For each $x_{0} \in X$ and $n \in \mathbb{N}$, put $x_{n+1}=T x_{n}$. It follows from (2.1) and Lemma 2 that

$$
\begin{aligned}
S\left(x_{n+1}, x_{n+1}, x_{n+2}\right)= & S\left(T x_{n}, T x_{n}, T x_{n+1}\right) \\
\leq & \phi\left(S\left(x_{n}, x_{n}, x_{n+1}\right), S\left(x_{n}, x_{n}, T x_{n}\right), S\left(x_{n+1}, x_{n+1}, T x_{n+1}\right),\right. \\
& \left.S\left(x_{n}, x_{n}, T x_{n+1}\right), S\left(x_{n+1}, x_{n+1}, T x_{n}\right)\right) \\
= & \phi\left(S\left(x_{n}, x_{n}, x_{n+1}\right), S\left(x_{n}, x_{n}, x_{n+1}\right), S\left(x_{n+1}, x_{n+1}, x_{n+2}\right),\right. \\
& \left.S\left(x_{n}, x_{n}, x_{n+2}\right), S\left(x_{n+1}, x_{n+1}, x_{n+1}\right)\right) \\
= & \phi\left(S\left(x_{n}, x_{n}, x_{n+1}\right), S\left(x_{n}, x_{n}, x_{n+1}\right), S\left(x_{n+1}, x_{n+1}, x_{n+2}\right),\right. \\
& \left.S\left(x_{n}, x_{n}, x_{n+2}\right), 0\right) .
\end{aligned}
$$

By condition $\left(C S M_{3}\right)$ and Lemma 2, we have

$$
\begin{aligned}
S\left(x_{n}, x_{n}, x_{n+2}\right) & \leq 2 S\left(x_{n}, x_{n}, x_{n+1}\right)+S\left(x_{n+2}, x_{n+2}, x_{n+1}\right) \\
& =2 S\left(x_{n}, x_{n}, x_{n+1}\right)+S\left(x_{n+1}, x_{n+1}, x_{n+2}\right) .
\end{aligned}
$$

Since $\phi$ satisfies the condition $\left(A_{1}\right)$, there exists $k \in[0,1)$ such that

$$
S\left(x_{n+1}, x_{n+1}, x_{n+2}\right) \leq k S\left(x_{n}, x_{n}, x_{n+1}\right) \leq k^{n+1} S\left(x_{0}, x_{0}, x_{1}\right) .
$$

Thus for all $n<m$, by using $\left(C S M_{3}\right)$, Lemma 2 and equation (2.4), we have

$$
\begin{aligned}
S\left(x_{n}, x_{n}, x_{m}\right) & \leq 2 S\left(x_{n}, x_{n}, x_{n+1}\right)+S\left(x_{m}, x_{m}, x_{n+1}\right) \\
& =2 S\left(x_{n}, x_{n}, x_{n+1}\right)+S\left(x_{n+1}, x_{n+1}, x_{m}\right) \\
& \cdots \\
& \leq 2\left[k^{n}+\cdots+k^{m-1}\right] S\left(x_{0}, x_{0}, x_{1}\right) \\
& \leq\left(\frac{2 k^{n}}{1-k}\right) S\left(x_{0}, x_{0}, x_{1}\right)
\end{aligned}
$$

This implies that

$$
\left\|S\left(x_{n}, x_{n}, x_{m}\right)\right\| \leq\left(\frac{2 k^{n} K}{1-k}\right)\left\|S\left(x_{0}, x_{0}, x_{1}\right)\right\| .
$$

Taking the limit as $n, m \rightarrow \infty$, we get

$$
\left\|S\left(x_{n}, x_{n}, x_{m}\right)\right\| \rightarrow 0
$$

since $0<k<1$. Thus, we have $S\left(x_{n}, x_{n}, x_{m}\right) \rightarrow 0$ as $n, m \rightarrow \infty$.

This shows that the sequence $\left\{x_{n}\right\}$ is a Cauchy sequence in the complete cone $S$-metric space $(X, S)$. By the completeness of the space, we have $\lim _{n \rightarrow \infty} x_{n}=x \in X$. Moreover, taking the limit as $m \rightarrow \infty$ we get

$$
S\left(x_{n}, x_{n}, x\right) \leq\left(\frac{2 k^{n+1}}{1-k}\right) S\left(x_{0}, x_{0}, x_{1}\right)
$$


It implies that

$$
S\left(T x_{n}, T x_{n}, x\right) \leq\left(\frac{2 k^{n}}{1-k}\right) S\left(x_{0}, x_{0}, T x_{0}\right)
$$

Now we prove that $x$ is a fixed point of $T$. By using inequality (2.1) again we obtain

$$
\begin{aligned}
S\left(x_{n+1}, x_{n+1}, T x\right)= & S\left(T x_{n}, T x_{n}, T x\right) \\
\leq & \phi\left(S\left(x_{n}, x_{n}, x\right), S\left(x_{n}, x_{n}, T x_{n}\right), S(x, x, T x),\right. \\
& \left.S\left(x_{n}, x_{n}, T x\right), S\left(x, x, T x_{n}\right)\right) \\
= & \phi\left(S\left(x_{n}, x_{n}, x\right), S\left(x_{n}, x_{n}, x_{n+1}\right), S(x, x, T x),\right. \\
& \left.S\left(x_{n}, x_{n}, T x\right), S\left(x, x, x_{n+1}\right)\right) .
\end{aligned}
$$

Note that $\phi \in \psi$, then using Lemma 3 and taking the limit as $n \rightarrow \infty$, we get

$$
S(x, x, T x) \leq \phi(0,0, S(x, x, T x), S(x, x, T x), 0) .
$$

Since $\phi$ satisfies the condition $\left(A_{1}\right)$, then $S(x, x, T x) \leq k .0=0$. This shows that $x=T x$. Thus $x$ is a fixed point of $T$.

(2) Let $x_{1}, x_{2}$ be fixed points of $T$. We shall prove that $x_{1}=x_{2}$. It follows from equation (2.1) and Lemma 2 that

$$
\begin{aligned}
S\left(x_{1}, x_{1}, x_{2}\right)= & S\left(T x_{1}, T x_{1}, T x_{2}\right) \\
\leq & \phi\left(S\left(x_{1}, x_{1}, x_{2}\right), S\left(x_{1}, x_{1}, T x_{1}\right), S\left(x_{2}, x_{2}, T x_{2}\right),\right. \\
& \left.S\left(x_{1}, x_{1}, T x_{2}\right), S\left(x_{2}, x_{2}, T x_{1}\right)\right) \\
= & \phi\left(S\left(x_{1}, x_{1}, x_{2}\right), S\left(x_{1}, x_{1}, x_{1}\right), S\left(x_{2}, x_{2}, x_{2}\right),\right. \\
& \left.S\left(x_{1}, x_{1}, x_{2}\right), S\left(x_{2}, x_{2}, x_{1}\right)\right) \\
= & \phi\left(S\left(x_{1}, x_{1}, x_{2}\right), 0,0, S\left(x_{1}, x_{1}, x_{2}\right), S\left(x_{2}, x_{2}, x_{1}\right)\right) \\
= & \phi\left(S\left(x_{1}, x_{1}, x_{2}\right), 0,0, S\left(x_{1}, x_{1}, x_{2}\right), S\left(x_{1}, x_{1}, x_{2}\right)\right) .
\end{aligned}
$$

Since $\phi$ satisfies the condition $\left(A_{2}\right)$, then $S\left(x_{1}, x_{1}, x_{2}\right)=0$. This shows that $x_{1}=x_{2}$. Thus the fixed point of $T$ is unique.

(3) Let $x$ be the fixed point of $T$ and $y_{n} \rightarrow x \in X$. By Lemma 4 we need to prove that 
$T y_{n} \rightarrow T x$. It follows from inequality (2.1) and Lemma 2 that

$$
\begin{aligned}
S\left(x, x, T y_{n}\right)= & S\left(T x, T x, T y_{n}\right) \\
\leq & \phi\left(S\left(x, x, y_{n}\right), S(x, x, T x), S\left(y_{n}, y_{n}, T y_{n}\right),\right. \\
& \left.S\left(x, x, T y_{n}\right), S\left(y_{n}, y_{n}, T x\right)\right) \\
= & \phi\left(S\left(x, x, y_{n}\right), S(x, x, x), S\left(y_{n}, y_{n}, T y_{n}\right),\right. \\
& \left.S\left(x, x, T y_{n}\right), S\left(y_{n}, y_{n}, x\right)\right) \\
= & \phi\left(S\left(x, x, y_{n}\right), 0, S\left(T y_{n}, T y_{n}, y_{n}\right),\right. \\
& \left.S\left(T y_{n}, T y_{n}, x\right), S\left(x, x, y_{n}\right)\right) .
\end{aligned}
$$

Since $\phi$ satisfies the condition $\left(A_{3}\right)$, by Lemma 2 and $\left(C S M_{3}\right)$, we have

$$
\begin{aligned}
S\left(T y_{n}, T y_{n}, y_{n}\right) & \leq 2 S\left(T y_{n}, T y_{n}, x\right)+S\left(y_{n}, y_{n}, x\right) \\
& =2 S\left(T y_{n}, T y_{n}, x\right)+S\left(x, x, y_{n}\right)
\end{aligned}
$$

then we have

$$
\begin{aligned}
S\left(x, x, T y_{n}\right) \leq & \phi\left(S\left(x, x, y_{n}\right), 0,0,0, S\left(x, x, y_{n}\right)\right) \\
& +\phi\left(0,0,2 S\left(T y_{n}, T y_{n}, x\right), S\left(T y_{n}, T y_{n}, x\right), 0\right) \\
\leq & \phi\left(S\left(x, x, y_{n}\right), 0,0,0, S\left(x, x, y_{n}\right)\right) \\
& +k S\left(T y_{n}, T y_{n}, x\right) \\
= & \phi\left(S\left(x, x, y_{n}\right), 0,0,0, S\left(x, x, y_{n}\right)\right) \\
& +k S\left(x, x, T y_{n}\right) . \quad(\text { by Lemma2) }
\end{aligned}
$$

Therefore

$$
S\left(x, x, T y_{n}\right) \leq\left(\frac{1}{1-k}\right) \phi\left(S\left(x, x, y_{n}\right), 0,0,0, S\left(x, x, y_{n}\right)\right)
$$

Note that $\phi \in \psi$, hence taking the limit as $n \rightarrow \infty$, we get $S\left(x, x, T y_{n}\right) \rightarrow 0$. This shows that $T y_{n} \rightarrow x=T x$. This completes the proof.

Next, we give some analogues of fixed point theorems in metric spaces for cone $S$-metric spaces by combining Theorem 1 with $\phi \in \psi$ and $\phi$ satisfies the conditions $\left(A_{1}\right),\left(A_{2}\right)$ and $\left(A_{3}\right)$. The following corollary is an analogue of Banach's contraction principle.

Corollary 1. Let $(X, S)$ be a complete cone $S$-metric space and $P$ be a normal cone with normal constant $K$. Suppose that the mapping $T: X \rightarrow X$ satisfies the following condition:

$$
S(T x, T x, T y) \leq h S(x, x, y)
$$


for all $x, y \in X$, where $h \in[0,1)$ is a constant. Then $T$ has a unique fixed point in $X$. Moreover, $T$ is continuous at the fixed point.

Proof. The assertion follows using Theorem 1 with $\phi(x, y, z, s, t)=h x$ for some $h \in[0,1)$ and all $x, y, z, s, t \in \mathbb{R}_{+}$.

The following corollary is an analogue of R. Kannan's result [10].

Corollary 2. Let $(X, S)$ be a complete cone $S$-metric space and $P$ be a normal cone with normal constant $K$. Suppose that the mapping $T: X \rightarrow X$ satisfies the following condition:

$$
S(T x, T x, T y) \leq q[S(x, x, T x)+S(y, y, T y)]
$$

for all $x, y \in X$, where $q \in\left[0, \frac{1}{2}\right)$ is a constant. Then $T$ has a unique fixed point in $X$. Moreover, $T$ is continuous at the fixed point.

Proof. The assertion follows using Theorem 1 with $\phi(x, y, z, s, t)=q(y+z)$ for some $q \in\left[0, \frac{1}{2}\right)$ and all $x, y, z, s, t \in \mathbb{R}_{+}$. Indeed, $\phi$ is continuous. First, we have $\phi(x, x, y, z, 0)=q(x+y)$. So, if $y \leq \phi(x, x, y, z, 0)$ with $z \leq 2 x+y$, then $y \leq\left(\frac{q}{1-q}\right) x$ with $\left(\frac{q}{1-q}\right)<1$. Thus, $T$ satisfies the condition $\left(A_{1}\right)$.

Next, if $y \leq \phi(y, 0,0, y, y)$, then $y=0$. Thus, $T$ satisfies the condition $\left(A_{2}\right)$.

Finally, if $x_{i} \leq y_{i}+z_{i}$ for $i \leq 5$, then

$$
\begin{aligned}
\phi\left(x_{1}, \ldots, x_{5}\right) & =q\left(x_{2}+x_{3}\right) \\
& \leq q\left[\left(y_{2}+z_{2}\right)+\left(y_{3}+z_{3}\right)\right] \\
& =q\left(y_{2}+y_{3}\right)+q\left(z_{2}+z_{3}\right) \\
& =\phi\left(y_{1}, \ldots, y_{5}\right)+\phi\left(z_{1}, \ldots, z_{5}\right) .
\end{aligned}
$$

Moreover

$$
\phi(0,0,2 y, y, 0)=q(0+2 y)=2 q y
$$

where $2 q<1$. Thus, $T$ satisfies the condition $\left(A_{3}\right)$.

The following corollary is an analogue of S. K. Chatterjae's result 4].

Corollary 3. Let $(X, S)$ be a complete cone $S$-metric space and $P$ be a normal cone with normal constant $K$. Suppose that the mapping $T: X \rightarrow X$ satisfies the following condition:

$$
S(T x, T x, T y) \leq p[S(x, x, T y)+S(y, y, T x)]
$$

for all $x, y \in X$, where $p \in\left[0, \frac{1}{2}\right)$ is a constant. Then $T$ has a unique fixed point in $X$. Moreover, $T$ is continuous at the fixed point. 
Proof. The assertion follows using Theorem 1 with $\phi(x, y, z, s, t)=p(s+t)$ for some $p \in\left[0, \frac{1}{2}\right)$ and all $x, y, z, s, t \in \mathbb{R}_{+}$. Indeed, $\phi$ is continuous. First, we have $\phi(x, x, y, z, 0)=p(z+0)$. So, if $y \leq \phi(x, x, y, z, 0)$ with $z \leq 2 x+y$, then $y \leq\left(\frac{2 p}{1-p}\right) x$ with $\left(\frac{2 p}{1-p}\right)<1$. Thus, $T$ satisfies the condition $\left(A_{1}\right)$.

Next, if $y \leq \phi(y, 0,0, y, y)=2 p y$, then $y=0$ since $p<\frac{1}{2}$. Thus, $T$ satisfies the condition $\left(A_{2}\right)$.

Finally, if $x_{i} \leq y_{i}+z_{i}$ for $i \leq 5$, then

$$
\begin{aligned}
\phi\left(x_{1}, \ldots, x_{5}\right) & =p\left(x_{4}+x_{5}\right) \\
& \leq p\left[\left(y_{4}+z_{4}\right)+\left(y_{5}+z_{5}\right)\right] \\
& =p\left(y_{4}+y_{5}\right)+p\left(z_{4}+z_{5}\right) \\
& =\phi\left(y_{1}, \ldots, y_{5}\right)+\phi\left(z_{1}, \ldots, z_{5}\right) .
\end{aligned}
$$

Moreover

$$
\phi(0,0,2 y, y, 0)=p(y+0)=p y
$$

where $p<1$. Thus, $T$ satisfies the condition $\left(A_{3}\right)$.

The following corollary is an analogue of S. Reich's result [21].

Corollary 4. Let $(X, S)$ be a complete cone $S$-metric space and $P$ be a normal cone with normal constant $K$. Suppose that the mapping $T: X \rightarrow X$ satisfies the following condition:

$$
S(T x, T x, T y) \leq a S(x, x, y)+b S(x, x, T x)+c S(y, y, T y)
$$

for all $x, y \in X$, where $a, b, c \geq 0$ are constants with $a+b+c<1$. Then $T$ has a unique fixed point in $X$. Moreover, if $c<\frac{1}{2}$, then $T$ is continuous at the fixed point.

Proof. The assertion follows using Theorem 1 with $\phi(x, y, z, s, t)=a x+b y+c z$ for some $a, b, c \geq 0$ are constants with $a+b+c<1$ and all $x, y, z, s, t \in \mathbb{R}_{+}$. Indeed, $\phi$ is continuous. First, we have $\phi(x, x, y, z, 0)=a x+b x+c y$. So, if $y \leq \phi(x, x, y, z, 0)$ with $z \leq 2 x+y$, then $y \leq\left(\frac{a+b}{1-c}\right) x$ with $\left(\frac{a+b}{1-c}\right)<1$. Thus, $T$ satisfies the condition $\left(A_{1}\right)$.

Next, if $y \leq \phi(y, 0,0, y, y)=a y$, then $y=0$ since $a<1$. Thus, $T$ satisfies the condition $\left(A_{2}\right)$.

Finally, if $x_{i} \leq y_{i}+z_{i}$ for $i \leq 5$, then

$$
\begin{aligned}
\phi\left(x_{1}, \ldots, x_{5}\right) & =a x_{1}+b x_{2}+c x_{3} \\
& \leq a\left(y_{1}+z_{1}\right)+b\left(y_{2}+z_{2}\right)+c\left(y_{3}+z_{3}\right) \\
& =\left(a y_{1}+b y_{2}+c y_{3}\right)+\left(a z_{1}+b z_{2}+c z_{3}\right) \\
& =\phi\left(y_{1}, \ldots, y_{5}\right)+\phi\left(z_{1}, \ldots, z_{5}\right) .
\end{aligned}
$$


Moreover

$$
\phi(0,0,2 y, y, 0)=a .0+b .0+c .2 y=2 c y
$$

where $2 c<1$. Thus, $T$ satisfies the condition $\left(A_{3}\right)$.

The following corollary is an analogue of L. B. Ćirić's result [5].

Corollary 5. Let $(X, S)$ be a complete cone $S$-metric space and $P$ be a normal cone with normal constant $K$. Suppose that the mapping $T: X \rightarrow X$ satisfies the following condition:

$$
\begin{gathered}
S(T x, T x, T y) \leq h \max \{S(x, x, y), S(x, x, T x), S(y, y, T y) \\
S(x, x, T y), S(y, y, T x)\}
\end{gathered}
$$

for all $x, y \in X$, where $h \in\left[0, \frac{1}{3}\right)$ is a constant. Then $T$ has a unique fixed point in $X$. Moreover, $T$ is continuous at the fixed point.

Proof. The assertion follows using Theorem 1 with $\phi(x, y, z, s, t)=h \max \{x$, $y, z, s, t\}$ for some $h \in\left[0, \frac{1}{3}\right)$ and all $x, y, z, s, t \in \mathbb{R}_{+}$. Indeed, $\phi$ is continuous. First, we have $\phi(x, x, y, z, 0)=h \max \{x, x, y, z, 0\}$. So, if $y \leq \phi(x, x, y, z, 0)$ with $z \leq 2 x+y$, then $y \leq h x$ or $y \leq h z \leq h(2 x+y)$. Then $y \leq k x$ with $k=\max \left\{h, \frac{2 h}{1-h}\right\}<1$. Thus, $T$ satisfies the condition $\left(A_{1}\right)$.

Next, if $y \leq \phi(y, 0,0, y, y)=h \max \{y, 0,0, y, y\}=h y$, then $y=0$ since $h<\frac{1}{3}$. Thus, T satisfies the condition $\left(A_{2}\right)$.

Finally, if $x_{i} \leq y_{i}+z_{i}$ for $i \leq 5$, then

$$
\begin{aligned}
\phi\left(x_{1}, \ldots, x_{5}\right) & =h \max \left\{x_{1}, \ldots, x_{5}\right\} \\
& \leq h \max \left\{y_{1}+z_{1}, \ldots, y_{5}+z_{5}\right\} \\
& \leq h \max \left\{y_{1}, \ldots, y_{5}\right\}+h \max \left\{z_{1}, \ldots, z_{5}\right\} \\
& =\phi\left(y_{1}, \ldots, y_{5}\right)+\phi\left(z_{1}, \ldots, z_{5}\right) .
\end{aligned}
$$

Moreover

$$
\phi(0,0,2 y, y, 0)=h \max \{0,0,2 y, y, 0\}=2 h y
$$

where $2 h<1$. Thus, $T$ satisfies the condition $\left(A_{3}\right)$.

Example 8. Let $E=\mathbb{R}^{2}$, the Euclidean plane, $P=\left\{(x, y) \in \mathbb{R}^{2}: x \geq 0, y \geq 0\right\}$ a normal cone in $E$ and $X=\mathbb{R}$. Then the function $S: X^{3} \rightarrow E$ defined by $S(x, y, z)=|x-z|+|y-z|$ for all $x, y, z \in X$. Then $(X, S)$ is a cone $S$-metric space. Now, we consider the mapping $T: X \rightarrow X$ by $T(x)=\frac{x}{2}$ and $\left\{x_{n}\right\}=\left\{\frac{1}{2^{n}}\right\}$ for all $n \in \mathbb{N}$ is a sequence converging to zero. 


\section{Result Analysis}

(1) Taking $x=x_{n-1}$ and $y=x_{n}$ in inequality (2.1) and using $\left(C S M_{3}\right)$, we have

$$
\begin{aligned}
S\left(x_{n}, x_{n}, x_{n+1}\right)= & S\left(T x_{n-1}, T x_{n-1}, T x_{n}\right) \\
\leq & \phi\left(S\left(x_{n-1}, x_{n-1}, x_{n}\right), S\left(x_{n-1}, x_{n-1}, T x_{n-1}\right), S\left(x_{n}, x_{n}, T x_{n}\right),\right. \\
& \left.S\left(x_{n-1}, x_{n-1}, T x_{n}\right), S\left(x_{n}, x_{n}, T x_{n-1}\right)\right) \\
= & \phi\left(S\left(x_{n-1}, x_{n-1}, x_{n}\right), S\left(x_{n-1}, x_{n-1}, x_{n}\right), S\left(x_{n}, x_{n}, x_{n+1}\right),\right. \\
& \left.S\left(x_{n-1}, x_{n-1}, x_{n+1}\right), S\left(x_{n}, x_{n}, x_{n}\right)\right) \\
= & \phi\left(S\left(x_{n-1}, x_{n-1}, x_{n}\right), S\left(x_{n-1}, x_{n-1}, x_{n}\right), S\left(x_{n}, x_{n}, x_{n+1}\right),\right. \\
& \left.S\left(x_{n-1}, x_{n-1}, x_{n+1}\right), 0\right) \\
\leq & \phi\left(S\left(x_{n-1}, x_{n-1}, x_{n}\right), S\left(x_{n-1}, x_{n-1}, x_{n}\right), S\left(x_{n}, x_{n}, x_{n+1}\right),\right. \\
& \left.2 S\left(x_{n-1}, x_{n-1}, x_{n}\right)+S\left(x_{n}, x_{n}, x_{n+1}\right), 0\right) .
\end{aligned}
$$

Since $\phi$ satisfies the condition $\left(A_{1}\right)$, so there exists $k \in[0,1)$ such that

$$
S\left(x_{n}, x_{n}, x_{n+1}\right) \leq k S\left(x_{n-1}, x_{n-1}, x_{n}\right)
$$

or

$$
\left.2\left(x_{n}-x_{n+1}\right)\right) \leq k \cdot 2\left(x_{n-1}-x_{n}\right)
$$

or

$$
\left.\left(\frac{1}{2^{n}}-\frac{1}{2^{n+1}}\right)\right) \leq k\left(\frac{1}{2^{n-1}}-\frac{1}{2^{n}}\right)
$$

or

$$
k \geq \frac{1}{2}
$$

If we take $0<k<1$, then inequality (2.1) is satisfied. Thus all the conditions of Theorem 1 are satisfied. Hence by Theorem 1, T has a unique fixed point. Here, note that ' 0 ' is the unique fixed point of $T$.

(2) Let $\left\{y_{n}\right\}=\left\{\frac{1}{3^{n}}\right\}$ be a sequence in $X$ converging to the fixed point $z=0$, then we have to show that $T y_{n} \rightarrow z$ as $n \rightarrow \infty$, that is, $T$ is continuous at the fixed point of $T$, we have

$$
\lim _{n \rightarrow \infty} T y_{n}=T\left(\lim _{n \rightarrow \infty} y_{n}\right)=T(0)=0=z
$$


That is,

$$
T y_{n} \rightarrow z \text { as } n \rightarrow \infty
$$

Thus, $T$ is continuous at the fixed point of $T$.

Example 9. Let $E=\mathbb{R}^{2}$, the Euclidean plane, $P=\left\{(x, y) \in \mathbb{R}^{2}: x \geq 0, y \geq 0\right\}$ a normal cone in $E$ and $X=\mathbb{R}$. Then the function $S: X^{3} \rightarrow E$ defined by $S(x, y, z)=|x-z|+|y-z|$ for all $x, y, z \in X$. Then $(X, S)$ is a cone $S$-metric space. Now, we consider the mapping $T: X \rightarrow X$ by $T(x)=\frac{x}{3}$. Then

$$
\begin{aligned}
S(T x, T x, T y) & =|T x-T y|+|T x-T y| \\
& =2|T x-T y|=2\left|\left(\frac{x}{3}\right)-\left(\frac{y}{3}\right)\right| \\
& =\frac{2}{3}|x-y| \\
& =\frac{1}{3}(2|x-y|) \\
& \leq \frac{1}{2}(2|x-y|) \\
& =h S(x, x, y)
\end{aligned}
$$

where $h=\frac{1}{2}<1$. Thus $T$ satisfies all the conditions of Corollary 1 and clearly $0 \in X$ is the unique fixed point of $T$.

\section{Conclusion}

In this paper, we establish some fixed point theorems using implicit relation in the framework of complete cone $S$-metric spaces. Our results extend, unify and generalize several results from the existing literature. Especially, they extend the corresponding results of Sedghi and Dung [24] from complete $S$-metric spaces to the setting of complete cone $S$-metric spaces. However, these results have vast potential in solving various nonlinear problems in functional analysis, differential and integral equations, computer science and engineering.

\section{Acknowledgement}

The author is grateful to the anonymous referees for their careful reading and valuable suggestions to improve the manuscript. 


\section{References}

[1] A. Aliouche and V. Popa, General common fixed point theorems for occasionally weakly compatible hybmappings and applications, Novi Sad J. Math. 39(1) (2009), 89-109.

[2] V. Berinde, Approximating fixed points of implicit almost contractions, Hacet. J. Math. Stat. 41 (2012), no. 1, 93-102.

[3] V. Berinde and F. Vetro, Common fixed points of mappings satisfying implicit contractive conditions, Fixed Point Theory Appl. 2012, 2012:105, 8 pp.

[4] S. K. Chatterjae, Fixed point theorems compactes, Rend. Acad. Bulgare Sci. 25 (1972), 727730.

[5] Lj. B. Ćirić, A generalization of Banach's contraction principle, Proc. Amer. Math. Soc. 45 (1974), 267-273.

[6] D. Dhamodharan and R. Krishnakumar, Cone $S$-metric space and fixed point theorems of contractive mappings, Annals of Pure Appl. Math. 14(2) (2017), 237-243.

[7] K. Deimling, Nonlinear functional analysis, Springer-Verlag, Berlin, 1985.

[8] L.-G. Huang and X. Zhang, Cone metric spaces and fixed point theorems of contractive mappings, J. Math. Anal. Appl. 332 (2007), no. 2, 1468-1476.

[9] M. Imdad, S. Kumar and M. S. Khan, Remarks on some fixed point theorems satisfying implicit relations, Rad. Mat. 11 (2002), no. 1, 135-143.

[10] R. Kannan, Some results on fixed point theorems, Bull. Calcutta Math. Soc. 60(1969), 71-78.

[11] J. K. Kim, S. Sedghi and N. Shobkolaei, Common fixed point theorems for the $R$-weakly commuting mappings in $S$-metric spaces, J. Comput. Anal. Appl. 19 (2015), no. 4, 751-759.

[12] R. Krishnakumar and D. Dhamodharan, Fixed point theorems in normal cone metric space, Int. J. Math. Sci. Engg. Appl. 10(III) (2016), 213-224.

[13] Nguyen Van Dung, N. T. Hieu and S. Radojević, Fixed point theorems for $g$-monotone maps on partially ordered $S$-metric spaces, Filomat 28 (2014), no. 9, 1885-1898.

[14] N. Yilmaz Özgür and N. Taş, Some fixed point theorems on $S$-metric spaces, Mat. Vesnik 69 (2017), no. 1, 39-52.

[15] V. Popa, Fixed point theorems for implicit contractive mappings, Stud. Cercet. Ştiinţ. Ser. Mat. Univ. Bacău No. 7 (1997), 127-133 (1999). 
[16] V. Popa, On some fixed point theorems for compatible mappings satisfying an implicit relation, Demonstr. Math. 32(1) (1999), 157-163.

[17] V. Popa, A general fixed point theorem for four weakly compatible mappings satisfying an implicit relation, Filomat No. 19 (2005), 45-51.

[18] V. Popa and A.-M. Patriciu, A general fixed point theorem for pairs of weakly compatible mappings in $G$-metric spaces, J. Nonlinear Sci. Appl. 5 (2012), no. 2, Special issue, 151-160.

[19] V. Popa and A.-M. Patriciu, Fixed point theorems for two pairs of mappings in partial metric spaces, Facta Univ. Ser. Math. Inform. 31 (2016), no. 5, 969-980.

[20] M. U. Rahman and M. Sarwar, Fixed point results of Altman integral type mappings in $S$-metric spaces, Int. J. Anal. Appl. 10(1) (2016), 58-63.

[21] S. Reich, Some remarks concerning contraction mappings, Canad. Math. Bull. 14 (1971), $121-124$.

[22] Sh. Rezapour and R. Hamlbarani, Some notes on the paper: "Cone metric spaces and fixed point theorems of contractive mappings" [J. Math. Anal. Appl. 332 (2007), no. 2, 1468-1476; by L.-G. Huang and X. Zhang, J. Math. Anal. Appl. 345 (2008), no. 2, 719-724.

[23] S. Sedghi, N. Shobe and A. Aliouche, A generalization of fixed point theorems in $S$-metric spaces, Mat. Vesnik 64 (2012), no. 3, 258-266.

[24] S. Sedghi and N. V. Dung, Fixed point theorems on S-metric space, Mat. Vesnik 66(1) (2014), $113-124$.

[25] S. Sedghi, N. Shobe and T. Došenović, Fixed point results in $S$-metric spaces, Nonlinear Funct. Anal. Appl. 20(1) (2015), 55-67.

[26] S. Sedghi et al., Common fixed point theorems for contractive mappings satisfying $\Phi$-maps in S-metric spaces, Acta Univ. Sapientiae Math. 8 (2016), no. 2, 298-311.

[27] N. Tas and N. Yilmaz Ozgur, New generalized fixed point results on $S_{b}$-metric spaces, arxiv:1703.01868v2 [math.gn] 17 apr. 2017.

[28] J. S. Vandergraft, Newton's method for convex operators in partially ordered spaces, SIAM J. Numer. Anal. 4 (1967), 406-432.

[29] P. P. Zabrejko, K-metric and $K$-normed linear spaces: survey, Collect. Math. 48 (1997), no. $4-6,825-859$. 